\title{
Successful strategies in repeated minority games
}

\author{
T. Chmura ${ }^{\mathrm{a}, \mathrm{b}}$, T. Pitz ${ }^{\mathrm{a}, \mathrm{b}, *}$ \\ ${ }^{a}$ Laboratory of Experimental Economics, University of Bonn, Adenauerallee 24-42, 53113 Bonn, Germany \\ ${ }^{\mathrm{b}}$ Physics of Transport and Traffic, University Duisburg-Essen, 47048 Duisburg, Germany
}

Received 5 September 2005; received in revised form 27 December 2005

Available online 25 January 2006

\begin{abstract}
The minority game is an important example of a non-cooperative $n$-person game, which can be applied on different situations with social and economic contexts. We analysed the minority game as an elementary traffic scenario in which human participants had to choose 100 times between a road $A$ and a road $B$. In each period, the road, which was chosen by the minority of players won. At first view, there seems to be no outstandingly advisable strategy for the participants to enhance their payoffs because both roads have the same properties. However, an important observation is that the number of road changes of a participant is negatively correlated to his/her cumulative payoff. On average, subjects with high numbers of road changes received less money than participants who stoically chose the same road. Furthermore one could increase the coordination of the players by providing the players distribution on both roads in the last period.
\end{abstract}

(C) 2006 Elsevier B.V. All rights reserved.

Keywords: Minority game; Laboratory experiments; Traffic behaviour

\section{Introduction}

The minority game is an important example of a $n$-person game with no pure equilibria and can be applied on different situations with social and economic contexts. The minority game, which is also called the El Farol Bar Problem, was introduced by Arthur [1] and theoretically analysed in detail by [2,3].

The rules of the minority game can be described in a short way: a number of agents $n$ have to choose in several periods whether to enter room A or B. Those agents who chose the less crowded room win whereas the others lose.

\section{Experimental setup}

For this paper, the minority problem was transferred to a route choice context. We did minority game experiments at the Laboratory of Experimental Economics at the University of Bonn. A number of experiments on route choice behaviour (e.g. [4,5]) were already reported. Here we focus on the route choice in

\footnotetext{
*Corresponding author. Present address: Shanghai Jiao Tong University, Antai School of Management, No. 535, Fa Hua Zhen Road, Shanghai, 200052, People's Republic of China.

E-mail addresses: chmura@uni-bonn.de (T. Chmura), tpitz@unni-bonn.de (T. Pitz).
} 
a generic two-route scenario, which already has been investigated in the literature (e.g. [6]). In Ref. [7] a volatile decision dynamics far from optimal payoff distribution was observed in route choice experiments. They showed that specific guidance strategies are able to increase the performance of all users by reducing overreaction and stabilizing the decision dynamics.

In Ref. [8], a model for the behaviour of drivers in a city for analysing the emergent collective behaviour was discussed. They found that in absence of information noise, inductive drivers turn out to behave better than random drivers for low car densities, while at high densities the opposite occurs.

Our aim is to present minority game experiments with a large number of periods and with sufficiently many independent observations for meaningful applications of non-parametric significance tests.

In these experiments, subjects were told that in each of the 100 periods, they had to make a choice between a road $A$ and road $B$ for travelling from $X$ to $Y$ (Fig. 1).

The number of subjects in each session was 9. They were told that the travel times $t_{A}$ and $t_{B}$ on $\operatorname{road} A$ and $B$ depended on the numbers $n_{A}$ and $n_{B}$ of participants choosing $A$ and $B$, respectively:

$$
t_{A}=1, \quad t_{B}=0 \Leftrightarrow n_{A}<n_{B} \text { and } t_{B}=1, \quad t_{A}=0 \Leftrightarrow n_{A}>n_{B} .
$$

The period payoff was $t_{A}$ if $A$ was chosen and $t_{B}$ if $B$ was chosen. The total payoff of a subject was the sum of all 100 period payoffs converted proportionally to money payoffs in Euro. Two treatments were investigated. In treatment I, each subject received information whether the last of his own choices was shared by the minority or majority of the participants. In treatment II, additional feedback was provided about the distribution of participants on both routes in the last period. Six sessions were run with treatments I and II, respectively. No further information was given to the subjects. It seems that especially in treatment $\mathrm{I}$, there is no outstandingly advisable strategy for the participants to enhance their payoffs because due to the symmetry of the game, each road has the same properties. However, one can see that in some cases, the number of road changes of participants has a significant effect on their cumulative payoff at the end of the experiment.

Fig. 2 shows the number of participants on $\operatorname{road} A$ as a function of time for a typical session of treatments I and II. The mean number of players on road $A$ is 4.5 in treatment I and 4.43 in treatment II. This was expected since due to the experimental setup, there is no preference for one road.

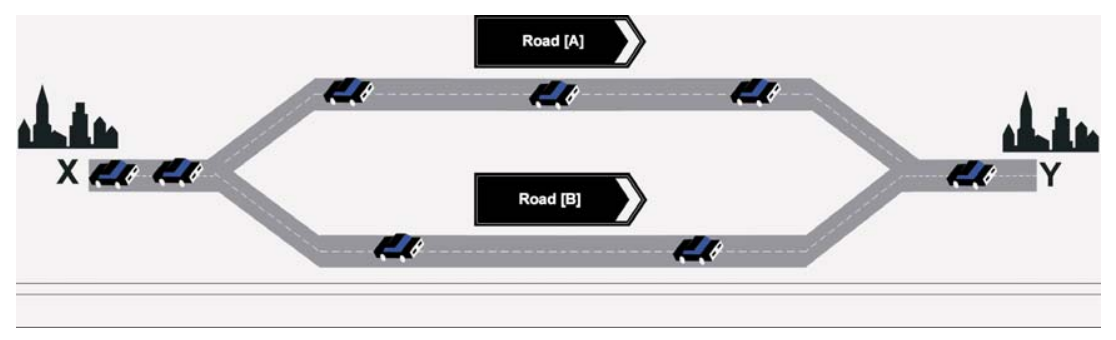

Fig. 1. Participants had to choose between a $\operatorname{road} A$ and a road $B$.

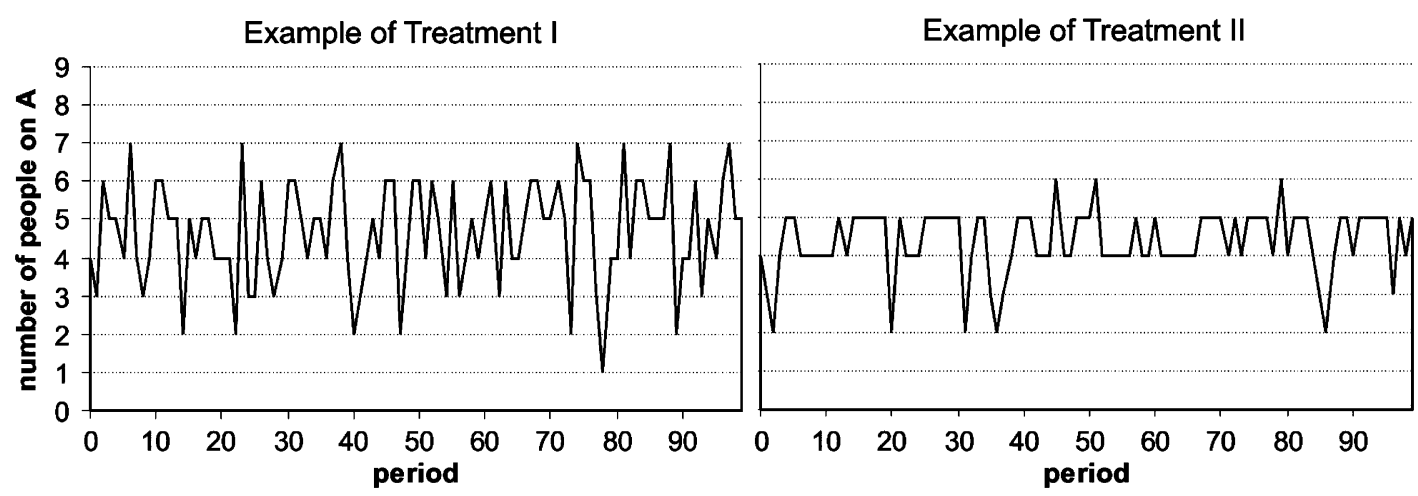

Fig. 2. Number of participants on $A$ : a typical session of treatments I and II. 


\section{Cumulative payoff, fluctuations and road changes}

The non-existence of pure strategy equilibria poses a coordination problem, which may be one of the reasons for non-convergence and the persistence of fluctuations in both treatments.

\subsection{Cumulative payoff}

The additional feedback in treatment II about the distribution of the players on both roads in the last period increases the cumulative payoff of participants. In Table 1 one can see for each treatment the average of cumulative payoff. The mean payoff in treatment II is significantly higher than in treatment I. The nullhypothesis can be rejected by a Wilcoxon-Mann-Whitney-Test on the significance level of $1 \%$ (one sided).

\subsection{Fluctuations}

Furthermore, the information in treatment II has a beneficial effect on the number of fluctuations, which is reduced. The fluctuations per period can be measured by the standard deviation of the number of participants choosing $A$ per period. The standard deviation of each treatment is listed in Table 1 . The fluctuations are obviously larger in treatment I than in treatment II. The effect is significant. The null-hypothesis is rejected by a Wilcoxon-Mann-Whitney-Test on the significance level of $1 \%$ (one sided).

\subsection{Road changes}

The reduction of fluctuations in treatment II could also be measured by the number of road changes per period. As one can see in Table 1, the mean number of road changes is significantly higher in treatment I. The null-hypothesis is rejected by the Wilcoxon-Mann-Whitney-Test on a level of $1 \%$ (one-sided).

In treatment II, subjects are informed about the distribution of players on both roads in the last period. This seems to be the reason for the greater number of road changes and also for the stronger fluctuations in treatment I. Feedback on the distribution of the players on both roads in the last period significantly reduces fluctuations and the number of road changes in treatment II compared to treatment I. The reason of the higher number of road changes and worse coordination of the players may be the lack of information in treatment I.

Table 1

Experimental results: treatments I and II

\begin{tabular}{|c|c|c|c|c|c|}
\hline & \multirow{2}{*}{$\frac{\text { Cumulative payoff }}{\text { Mean }}$} & \multicolumn{2}{|c|}{ Number of players on $A$} & \multirow{2}{*}{$\begin{array}{l}\text { Number of road changes } \\
\text { Mean }\end{array}$} & \multirow{2}{*}{$\begin{array}{l}\text { Road changes vs. cumulative payoff } \\
\text { Spearman rank correlation }\end{array}$} \\
\hline & & Mean & Std. dev. & & \\
\hline \multicolumn{6}{|l|}{ Treatment I } \\
\hline Session I 01 & 37.33 & 4.33 & 1.36 & 5.08 & -0.48 \\
\hline Session I 02 & 36.22 & 4.74 & 1.50 & 3.87 & 0.34 \\
\hline Session I 03 & 36.67 & 4.41 & 1.50 & 5.16 & -0.44 \\
\hline Session I 04 & 38.00 & 4.40 & 1.31 & 5.19 & -0.70 \\
\hline Session I 05 & 37.67 & 4.65 & 1.33 & 5.28 & -0.18 \\
\hline Session I 06 & 38.44 & 4.44 & 1.28 & 4.35 & -0.18 \\
\hline Treatment I & 37.89 & 4.50 & 1.38 & 4.82 & -0.27 \\
\hline \multicolumn{6}{|l|}{ Treatment II } \\
\hline Session II 01 & 37.89 & 4.19 & 1.35 & 3.99 & -0.51 \\
\hline Session II 02 & 38.89 & 4.62 & 1.19 & 3.68 & -0.54 \\
\hline Session II 03 & 40.78 & 4.36 & 1.05 & 3.67 & -0.30 \\
\hline Session II 04 & 41.22 & 4.34 & 0.97 & 5.19 & -0.82 \\
\hline Session II 05 & 42.44 & 4.62 & 0.84 & 4.67 & -0.27 \\
\hline Session II 06 & 43.33 & 4.50 & 0.67 & 4.44 & -0.78 \\
\hline Treatment II & 40.76 & 4.44 & 1.01 & 4.27 & -0.54 \\
\hline
\end{tabular}



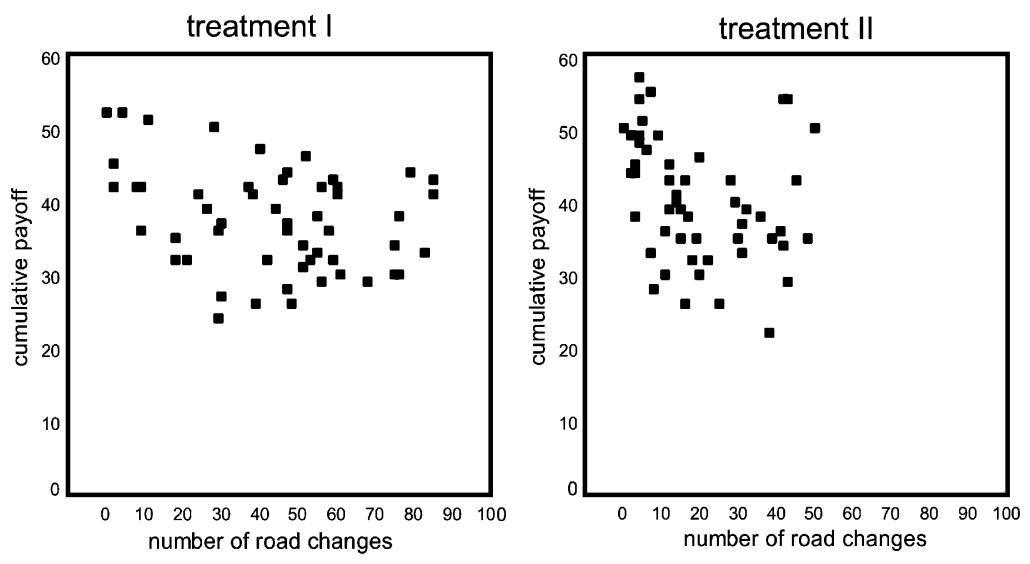

Fig. 3. Scatter diagram of cumulative payoff/number of road changes: treatments I and II.

With the additional information in treatment II the subjects could for example estimate the mean number of road changes per period and therefore determine more precisely the behaviour of the other players.

\section{Negative correlations between cumulative payoffs and road changes}

In all sessions except one of treatment I, the number of road changes of a subject is negatively correlated with the subject's payoff. Fig. 3 shows the negative correlation between the payoff and the number of road changes.

This effect could also be seen by calculating the Spearman rank correlation between cumulative payoffs and the number of road changes for each treatment as shown in Table 1. The mean number of the Spearman rank correlation coefficients in treatment $\mathrm{I}$ is -.27 and in treatment II -.54 . In 11 observations of both the treatments, the Spearman rank correlations between cumulative payoffs and the number of road changes are negative. This is a significant negative correlation between the total number of road changes and the size of fluctuations.

On average, subjects with high numbers of road changes received less money than participants who stoically chose the same road. In treatment II, this effect is stronger than in treatment I. In treatment I, road changes may serve the purpose of gathering information about the behaviour of the other players. This motivation has no basis in treatment II because each participant is informed about the distribution of players in former periods. However, road changes may also be attempted to improve payoffs. The finding of a negative correlation between a subject's payoff and the number of road changes suggests that on average, such attempts are not successful.

\section{References}

[1] W.B. Arthur, Inductive reasoning and bounded rationality, Am. Econ. Rev. 84 (1994) 406.

[2] D. Challet, Y.-C. Zhang, Emergence of cooperation and organization in an evolutionary game, Physica A 246 (1997) $407-418$.

[3] D. Challet, Y.-C. Zhang, On the minority game: analytical and numerical studies, Physica A 256 (1998) 514-532.

[4] P. Bonsall, The influence of route guidance advice on route choice in urban networks, Transportation 19 (1992) 1-23.

[5] H.S. Mahmassani, Y.H. Liu, Dynamics of commuting decision behaviour under advanced traveller information systems, Transp. Res. C 7 (1999) 91-107.

[6] Y. Iida, T. Akiyama, T. Uchida, Experimental analysis of dynamic route choice behaviour, Transp. Res. B 26 (1992) 17-32.

[7] D. Helbing, M. Schönhof, D. Kern, Volatile decision dynamics: experiments, stochastic description, intermittency control and traffic optimization, New J. Phys. 4 (2002) 33.

[8] A. De Martino, M. Marsili, R. Mulet, Adaptive drivers in a model of urban traffic, Europhys. Lett. 65 (2004) 283. 